\title{
H INDEX SCHOLAR: EL ÍNDICE H DE LOS PROFESORES DE LAS UNIVERSIDADES PÚBLICAS ESPAÑOLAS EN HUMANIDADES Y CIENCIAS SOCIALES
}

\author{
Emilio Delgado-López-Cózar, Enrique Orduña-Malea, Evaristo Jiménez- \\ Contreras y Rafael Ruiz-Pérez
}
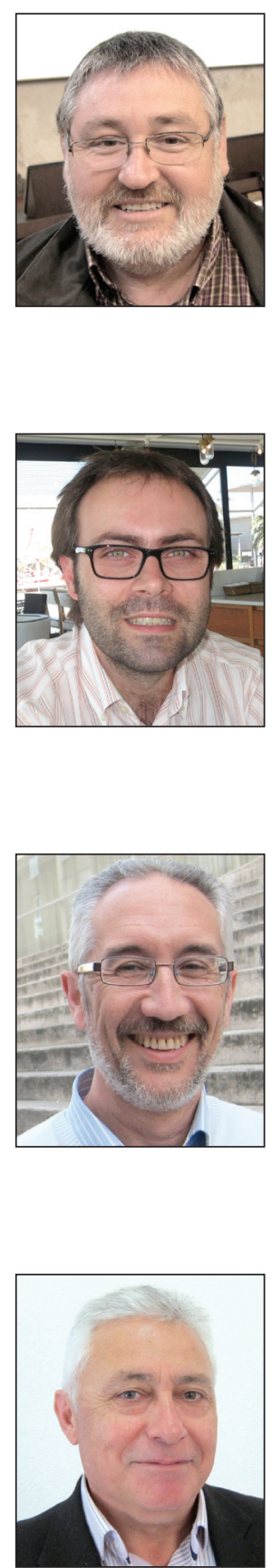

Emilio Delgado-López-Cózar, es catedrático de metodología de la investigación en la Facultad de Comunicación y Documentación de la Universidad de Granada. Miembro del Grupo de investigación $E C 3$, es creador de numerosos sistemas y herramientas para la evaluación científica como In-Recs, In-Recj, In-Rech, Metaranking EC3 de universidades españolas, Índice $h$ de las revistas españolas de CC SS y jurídicas, Índice $h$ de las revistas españolas según Google Scholar Metrics, Científica CIRC (Clasificación Integrada de Revistas Científicas) y DNA (Directorio Nacional de Autores). Es cofundador de la spin-off de asesoría científica EC3metrics. Estudia la evaluación de las revistas científicas, la ciencia, la investigación en documentación y el rendimiento científico de instituciones e investigadores*.

http://orcid.org/0000-0002-8184-551X

edelgado@ugr.es

Enrique Orduña-Malea, investigador posdoctoral en el DInstituto de Diseño y Fabricación (IDF) de la Universidad Politécnica de Valencia (UPV), es ingeniero técnico de telecomunicaciones, licenciado en documentación, master en contenidos multicanal y doctor en documentación (tesis doctoral en cibermetría) por la UPV. Desde 2012 es miembro del Grupo de investigación EC3 de la Universidad de Granada. Sus líneas de investigación se centran fundamentalmente en la cibermetría, tanto descriptiva (testeo de indicadores de naturaleza web y unidades de análisis) como instrumental (análisis de fuentes y buscadores) y aplicada (principalmente a entornos académicos)**. http://orcid.org/0000-0002-1989-8477

riorma@gmail.com

Evaristo Jiménez-Contreras es catedrático de bibliometría en la Facultad de Comunicación y Documentación de la Universidad de Granada, y director del Grupo de investigación EC3 (Evaluación de la Ciencia y de la Comunicación Científica). Sus líneas de investigación se centran en la evaluación de la actividad científica con metodología bibliométrica. Es uno de los promotores de los índices de impacto de las revistas españolas de ciencias sociales, jurídicas y humanas In-Recs, In-Recj, InRech, CIRC (Clasificación integrada de revistas científicas), Metaranking EC3 y Científica*. http://orcid.org/0000-0001-5668-7057

evaristo@ugr.es

Rafael Ruiz-Pérez es catedrático de documentación en la Facultad de Comunicación y Documentación de la Universidad de Granada y miembro del Grupo de investigación EC3 (Evaluación de la Ciencia y de la Comunicación Científica). Sus líneas de investigación y publicación están centradas en la evaluación de revistas científicas y en la normalización de nombres de entidades y autores. Es uno de los promotores de los índices de impacto de las revistas españolas de ciencias sociales, jurídicas y humanas In-Recs, In-Recj, In-Rech y DNA (Directorio nacional de autores)*. http://orcid.org/0000-0002-2656-1645

rruiz@ugr.es

* Universidad de Granada, EC3 Research Group Campus Cartuja. 18071 Granada, España

**Universidad Politécnica de Valencia, Instituto de Diseño y Fabricación, EC3 Research Group Camí de Vera, s/n. 46022 Valencia, España 


\title{
Resumen
}

$H$ Index Scholar es un índice bibliométrico sobre la productividad e impacto científico de la producción académica de los profesores e investigadores de universidades públicas españolas en humanidades y ciencias sociales. Se realiza mediante el recuento de sus publicaciones y de las citas bibliográficas que han recibido en Google Scholar. Se describen las principales funciones y características del producto. A pesar de los problemas técnicos y metodológicos que pueda presentar Google Scholar como fuente de información, los autores estiman que no afectan en lo sustancial a los índices h y g ofrecidos, estando dentro de una tasa de error del 10\%. La población total analizada ha sido de 40.993 profesores, de los que se visualiza un total de 13.518 situados en el primer tercil de sus respectivas áreas.

\section{Palabras clave}

Índice h, H Index Scholar, Google Scholar, Recuento de citas, Profesores universitarios, Universidades públicas, España, Humanidades, Ciencias sociales, Bibliometría, Rendimiento científico, Publicación científica.

Title: H Index Scholar: the h-index for Spanish public universities' professors of humanities and social sciences

\begin{abstract}
The $\mathrm{H}$-Index Scholar is a bibliometric index that measures the productivity and scientific impact of the academic production in humanities and social sciences by professors and researchers at public Spanish universities. The methodology consisted of counting their publications and citations received in Google Scholar. The main features and characteristics of the index are explained. Despite technical and methodological problems that Google Scholar might have as a source of information, the authors estimate that they do not affect substantially the calculated $\mathrm{h}$ and $\mathrm{g}$ indexes, probably being the error lower than $10 \%$. The total population analyzed was 40,993 researchers, but data are displayed only for 13,518 researchers, the ones located in the first tertile of their respective areas.
\end{abstract}

\section{Keywords}

H-Index, H Index Scholar, Google Scholar, Citation count, University professors, Public universities, Spain, Humanities, Social sciences, Bibliometrics, Scientific performance, Scientific publication.

Delgado-López-Cózar, Emilio; Orduña-Malea, Enrique; Jiménez-Contreras, Evaristo; Ruiz-Pérez, Rafael (2014). “H Index Scholar: el índice h de los profesores de las universidades públicas españolas en humanidades y ciencias sociales". El profesional de la información, enero-febrero, v. 23, n. 1, pp. 87-94.

http://dx.doi.org/10.3145/epi.2014.ene.11

\section{Justificación}

Se presenta $H$ Index Scholar ${ }^{1}$, un índice bibliométrico en abierto que pretende indagar sobre las tasas de productividad e impacto científico de la producción académica de los profesores e investigadores de universidades públicas españolas en humanidades y ciencias sociales, a partir del recuento de sus publicaciones -y de las citas bibliográficas que éstas han recibido- a través de Google Scholar.

La base de datos completa la constituyen (a fecha de julio de 2013) 40.993 profesores, aunque el producto final ofrece sólo el índice h de 13.518 profesores de universidades públicas españolas, agrupados en sus respectivas áreas de conocimiento (en concreto, el primer tercio de los autores con mayor índice h en cada área). En todo caso se debe mencionar que los cálculos originales se han aplicado a más de 50.000 profesores, población aproximada del personal que conforma las áreas de conocimiento de humanidades y ciencias sociales en España².

\subsection{Limitaciones en la evaluación de la investigación en humanidades y ciencias sociales}

La medición de la producción y el impacto de las publicaciones científicas, que son el principal medio empleado por los científicos para dar a conocer los resultados de sus crea- ciones (Cronin, 1984; Moed, 2005), se ha convertido en el instrumento por excelencia para determinar la relevancia e importancia y, subsidiariamente, la calidad de la actividad científica.

Sin embargo, mientras los indicadores bibliométricos fueron adoptados inmediatamente como herramientas evaluativas válidas en las disciplinas científico-técnicas (Callon et al., 1995), estos indicadores quedaron relegados a un segundo plano -cuando no abiertamente rechazados- en las humanidades y ciencias sociales. Las razones no son otras que la propia idiosincrasia de la investigación en estos campos así como la inexistencia de sistemas de información apropiados (Ardanuy, 2013).

Las peculiaridades de los investigadores de humanidades y ciencias sociales en sus prácticas de comunicación científica, estudiadas ampliamente, entre otros, por Broadus (1971), Hicks y Wang (1999), Hicks (2004), Nederhof (2006), Ardanuy (2009; 2013) y Archambault y Larivière (2010), se pueden sintetizar en las siguientes características:

- Publicar en una amplia variedad de medios, aunque con una especial predilección por las monografías: libros y capítulos de libros (Delgado-López-Cózar; Ruiz-Pérez, 2009).

- Utilizar su lengua vernácula como principal medio de expresión. 
- Publicar fundamentalmente en medios nacionales, bien sean revistas, actas de congresos o editoriales de monografías.

- Citar fundamentalmente monografías, trabajos publicados en su lengua nativa $y$ en medios nacionales (White et al., 2009).

Estas prácticas hacen casi inservibles las bases de datos bibliográficas tradicionales que ofrecen tanto recuentos de publicaciones como de citas (Web of science, Scopus, Psyclnfo, etc.). Dichas bases de datos cubren casi exclusivamente artículos de revistas, se orientan fundamentalmente a las disciplinas científico-técnicas y poseen un marcado sesgo anglosajón en cuanto a la procedencia e idioma de los documentos que indizan (Archambault et al., 2006), por lo que no resultan adecuadas dados los hábitos de publicación y citación de los investigadores en ciencias humanas y sociales (Osca-Lluch et al., 2013).

Tanto la mejora en los procesos de producción y evaluación de las monografías (Giménez-Toledo; Román-Román, 2009) como la reciente aparición de nuevas fuentes formales de citaciones orientadas a estos productos, muy especialmente el Book Citation Index (Leydesdorff; Felt, 2012), intentan paliar en parte estos problemas. Sin embargo, la todavía escasa representación actual de países y lenguas (más allá del entorno anglosajón) en este nuevo índice impide resolver los problemas derivados del sesgo idiomático (Torres-Salinas; Delgado-López-Cózar, 2013; Gorraiz et al., 2013).

\subsection{Google Scholar y la comunicación científica en la Web}

Sin embargo, la irrupción de internet a partir de la década de los noventa del siglo XX ha desencadenado una serie de cambios tecnológicos y metodológicos que han derivado en la creación de una serie de herramientas, como Google Scholar (Torres-Salinas et al., 2008) y Google Books (Kousha et al., 2011), que han abierto a su vez enormes posibilidades para la medición del rendimiento científico en las disciplinas humanísticas y sociales. Estos cambios son, de manera resumida, los siguientes:

Espectacular crecimiento de la bibliografía académica en acceso abierto en internet

El número de documentos científicos circulantes en la Red y en acceso abierto crece de manera muy rápida gracias a la conjunción de distintos fenómenos. En primer lugar, debido a la proliferación de sitios web personales e institucionales (grupos de investigación, departamentos, institutos), a través de las cuales los científicos y las entidades en que éstos trabajan intentan difundir los productos de su actividad académica (Thelwall, 2002; Orduña-Malea; Ontalba-Ruipérez, 2013). En segundo lugar, gracias a la multiplicación de repositorios institucionales y temáticos nacidos al calor del movimiento open access (Aguillo et al., 2010). Y en tercer lugar, por la presencia en la Web de catálogos de editoriales, bibliotecas, repertorios bibliográficos y directorios de publicaciones científicas de todas clases (Jacsó, 2008a).

Extraordinario crecimiento y mejora en la indización de la bibliografía científica a través de los buscadores

El nacimiento a finales de 2004 de Google Scholar marcó una auténtica revolución en la búsqueda, recuperación y acceso universal a las publicaciones científicas (Torres-Salinas et al., 2009; Orduña-Malea et al., 2009). Desde un primer momento, Google Scholar se convirtió no sólo en un buscador de documentos académicos sino también de las citas que éstos reciben (Jacsó, 2012). Todos los estudios realizados hasta el momento han puesto de relieve que Google Scholar cubre muchísimos más documentos que cualquiera de los sistemas de información científica tradicionales, indiza mayor variedad de géneros documentales en todos los idiomas sin restricción y recupera un porcentaje muy elevado de citas, muchas de las cuales proceden de las fuentes de referencia académica para el universo de las humanidades y ciencias sociales (Jacsó, 2008b; Kousha; Thelwall, 2007).

\section{Creación de nuevas herramientas}

Para la búsqueda masiva, el tratamiento y la medición de la información bibliográfica indizada por Google Scholar, de los cuales el programa "Publish or Perish"2 es el más representativo.

\section{Creación de índices bibliométricos}

Como el índice $h$, simples y sencillos de construir y entender y capaces de sintetizar las dos dimensiones de la actividad científica: producción e impacto. 


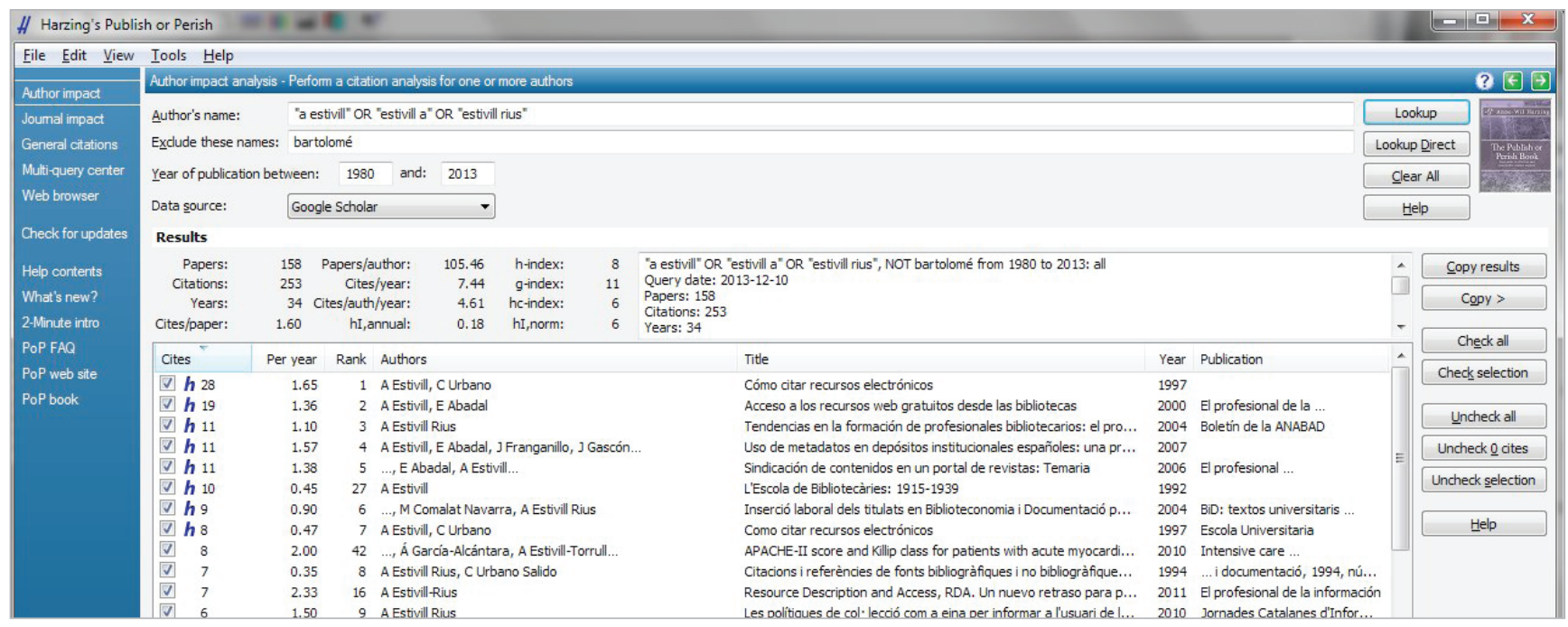

Figura 2. Interfaz de consulta de Publish or perish

En definitiva, Google Scholar se ha convertido ya (y teniendo en cuenta su ritmo de crecimiento vertiginoso, lo será más en el futuro inmediato), en una mina de información científica prodigiosa. Al rastrear en la Web toda la variopinta tipología de documentos de corte académico generados por los profesores en su actividad cotidiana (libros, capítulos, artículos de revistas científicas, material docente, tesis, ponencias y comunicaciones en congresos, informes, etc.), y al hacerlo en todos países e idiomas, deviene en una herramienta de suma utilidad para medir la producción y el impacto académico en el sentido más amplio del término (Kousha; Thelwall, 2008). Pero es especialmente de utilidad para los científicos de humanidades y ciencias sociales, pues controla como nadie lo ha hecho hasta ahora la bibliografía no anglosajona y aquella transmitida por medios distintos a las revistas científicas, que es la peor controlada por los sistemas de información dominantes en el mundo académico.

A pesar de los problemas técnicos y metodológicos que pueda presentar Google Scholar como fuente de información, tales como errores en la identificación de documentos y citas, falta de transparencia en la selección de fuentes, carencia de control y normalización de sus registros (Jacsó, 2008a; 2012), y a que desconozcamos con precisión y no controlemos sus entrañas (algo, por otra parte, casi imposible dada la naturaleza universal de la empresa), es tan masiva y rica la información académica que procesa que no podemos desaprovecharla a la hora de realizar análisis métricos de la información científica y académica en humanidades y ciencias sociales, campos inéditos hasta el momento.

A efectos evaluativos, ofrece luz donde antes había sombras, pues con ese motor de búsqueda se llega a lugares antes insospechados para ofrecer retratos que, aunque a veces sean de brocha gorda, son mejores que la nada.

Y esta afirmación adquiere todavía mayor verosimilitud e importancia en el caso de España, país que tiene un especial protagonismo en la circulación de información académica en la Web, como se pone de manifiesto en su preeminente presencia en Doar (Directory of open access repositories) ${ }^{4}$ y en el Ranking web de repositorios del mundo ${ }^{5}$.

\section{H Index Scholar}

\subsection{Objetivos}

En esta breve descripción del producto se desea especialmente subrayar el carácter experimental del mismo, dejando claros cuáles son los objetivos que pretende cubrir:

- Comprobar la capacidad de Google Scholar para recuperar la producción científica y académica de científicos de humanidades y ciencias sociales, invisibles a las tradicionales bases de datos por sus peculiares hábitos de publicación.

- Examinar su idoneidad para identificar la producción de entornos geográficos nacionales distintos al mundo anglosajón, y que usan lenguas de expresión distintas al inglés

- Determinar la fiabilidad y validez de los índices h y g, basados en información suministrada por Google Scholar, para detectar el núcleo de académicos de mayor influencia en las distintas disciplinas humanísticas y sociales.

El carácter experimental de esta acción se dirige asimismo a comprobar el grado de aceptación de productos de esta naturaleza en una comunidad académica, como es la de los profesores de los campos humanísticos y sociales, tan alejada de ejercicios y prácticas bibliométricas de este calibre. Y para ello, nada mejor que mostrar los listados resultantes en cada área de conocimiento para así analizar y evaluar las reacciones producidas.

En definitiva, este nuevo sistema es un hito más de la línea de investigación en la que está embarcado el grupo de investigación EC3 (Evaluación de la ciencia y de la comunicación científica $)^{6}$, desde hace unos años dirigida a descubrir las potencialidades de Google Scholar para la evaluación científica; y teniendo como telón de fondo la finalidad última de mejorar el acceso abierto al conocimiento en España, provocando la reacción de los académicos y científicos que ante iniciativas de este tipo procurarán aumentar su visibilidad e impacto subiendo su producción académica al espacio Web y, subsidiariamente, mejorando las herramientas de evaluación científica (indización exhaustiva y precisa en Google Scholar y en sus productos derivados). 


\subsection{Metodología}

$H$ Index Scholar emplea dos indicadores bibliométricos para medir la relevancia de la producción científica de un investigador:

- El índice h, propuesto por Jorge E. Hirsch (2005).

- El índice g, propuesto por Leo Egghe (2006).

El cálculo de los índices h y g se ha realizado a partir de la producción científica y las citas asociadas a la misma localizadas en Google Scholar. Se ha hecho una búsqueda bibliográfica de cada profesor, que contempla las principales variantes de su nombre, a fin de identificar todas sus posibles publicaciones y las citas enlazadas a las mismas. Tras un proceso manual de depuración de resultados (eliminación de duplicados y unión de citaciones), se calcula el índice $\mathrm{h}$ y g de cada autor. Se ha empleado el software Publish or perish diseñado por Anne-Wil Harzing (figura 2).

Los resultados ofrecidos se refieren de manera exclusiva a la fecha en la que se efectuaron las búsquedas bibliográficas (fechas que son indicadas expresamente en la cabecera de cada uno de los listados de las áreas de conocimiento consideradas), y no a los valores que actualmente los profesores puedan disponer.

Los índices se muestran por áreas de conocimiento (figura 3), que se agrupan en disciplinas científicas (figura 4), dentro de las cuatro principales ramas del conocimiento analizadas (ciencias sociales, ciencias jurídicas, ciencias humanas y arte). En cada uno de los listados sólo aparecen aquellos profesores que se encuentran en el núcleo de mayor rendimiento, tomando como umbral de corte el primer tercio de los profesores con mayor índice $\mathrm{h}$ de un área de conocimiento (a igualdad de h, se considera el valor logrado en el índice g).
Para adscribir a los profesores a sus áreas de conocimiento respectivas se han consultado los sitios web de todos los departamentos de todas las universidades públicas españolas de humanidades y ciencias sociales. En algunos casos, se han rastreado también sitios web de facultades, escuelas e institutos universitarios. Sin embargo, dadas las carencias de información en algunas de estas fuentes universitarias (especialmente departamentos) no siempre se ha podido realizar esta tarea exitosamente (Orduña-Malea, 2012).

En los casos en que un departamento está compuesto por más de un área de conocimiento y la fuente no permite dis-

\section{Ciencias sociales}

\begin{tabular}{|c|c|c|c|c|c|c|}
\hline $\begin{array}{l}\text { Clencias } \\
\text { Sociales }\end{array}$ & $\begin{array}{l}\text { Ciencias } \\
\text { Juridicas }\end{array}$ & $\begin{array}{l}\text { Ciencias } \\
\text { Humanas }\end{array}$ & Arte & $\leftarrow$ & & \\
\hline \multicolumn{3}{|c|}{ ECONOMIA } & EDUCA & & PSICOLOGÍA & SOCIOLOGIAA \\
\hline \multicolumn{3}{|c|}{ Comercialización e Inv. de Mercados } & Didáctic & Organización Escolar & Metodología Cc. del Comportamiento & Sociología \\
\hline \multicolumn{3}{|c|}{ Fundamentos del Análisis Económico } & Didáctic & la Expresión Corporal & Personalidad, Evaluación y Trat. Psicológico & Trabajo Social y Servicios Sociales \\
\hline \multicolumn{3}{|c|}{ Mét. cuantitativos para Eco. y Emp. } & Didáctic & la Expresión Musical & Psicobiología & Sin adscribir a área de conocimiento \\
\hline \multicolumn{3}{|c|}{ Organización de Empresas } & Didáctic & la Expresión Plástica & Psicología Básica & COMUNICACIÓN \\
\hline \multicolumn{3}{|c|}{ Economía Aplicada } & Didáctic & la Lengua y Literatura & Psicologia Evolutiva y de la Educación & Com. Audiovisual y Publicidad \\
\hline \multicolumn{3}{|c|}{ Economía Financiera y Contabilidad } & Didáctic & la Matemática & Psicologia Social & Periodismo \\
\hline \multicolumn{3}{|c|}{ Economía, Sociología y Política Agraria } & Didáctic & las Ciencias Exp. & Sin adscribir a área de conocimiento & Sin adscribir a área de conocimiento \\
\hline \multicolumn{3}{|c|}{ Sin adscribir a área de conocimiento } & Didáctic & las Ciencias Sociales & & DOCUMENTACIÓN \\
\hline \multicolumn{3}{|c|}{ GEOGRAFIA } & Educaci & Fisica y Deportiva & & ANTROPOLOGIAA \\
\hline \multicolumn{3}{|c|}{ Geografia Fisica } & Mét. In & Diagnóstico en Educación & & Antropología \\
\hline \multicolumn{3}{|c|}{ Geografia Humana } & Teoría & storia de la Educación & & Sin adscribir a área de conocimiento \\
\hline \multicolumn{3}{|c|}{$\begin{array}{l}\text { Análisis Geográfico Regional } \\
\text { Urbanistica y Ordenación Territorio }\end{array}$} & Sin ads & ir a área de conocimiento & & $\begin{array}{l}\text { CIENCIA POLITICA Y DE LA } \\
\text { ADMINISTRACIÓN }\end{array}$ \\
\hline Sin adscri & a área de co & ocimiento & & & & \\
\hline
\end{tabular}

criminar a qué área están adscritos los profesores, éstos se han agrupado en un apartado denominado "Sin adscribir a área de conocimiento". Se proporciona de manera adicional un formulario de consulta ${ }^{7}$ para que las universidades, áreas y departamentos en los que no ha sido factible realizar dicha adscripción, puedan proporcionar esta información directamente.

Figura 4. Ejemplo de áreas y disciplinas, dentro de ciencias sociales 


\subsection{Resultados}

En la tabla 1 se muestra la cantidad total de profesores analizados (puesto que en $H$ Index Scholar sólo se muestran los profesores en el primer tercil de cada área, la tabla 1 incluye tanto los profesores calculados, como los finalmente visualizados).

En el caso de ciencias jurídicas y arte, se muestran directamente las áreas de conocimiento, sin embargo, en el caso de ciencias sociales y humanas, dada la gran cantidad de áreas (35 en ciencias sociales y 34 en humanas), se han agrupado en disciplinas.

Los datos muestran la preponderancia de estos dos grandes campos, especialmente los profesores adscritos a disciplinas como economía (8.169), educación (5.671), psicología (3.874) e historia (3.546), que juntos suponen el 51,9\% de la población total analizada (40.993 profesores, de los que finalmente se visualiza un total de 13.518 profesores, situados en el primer tercil de sus áreas correspondientes).

Por otro lado, las ciencias jurídicas (6.445 profesores) y, sobre todo, las artísticas (966), representan fracciones más reducidas de profesorado, no llegando ninguna de las áreas que componen estas disciplinas a superar el millar de profesores.

Como se indica en la metodología, los profesores analizados son aquellos cuyas sedes web departamentales ofrecían en el momento del análisis información sobre la correcta adscripción a áreas o, al menos, información sobre el profesorado perteneciente al departamento. Por ello, estos datos representan de manera indirecta la calidad de la información académica que las universidades públicas españolas están ofreciendo en sus webs departamentales.

\section{Discusión y conclusiones}

Puesto que es la primera vez que se aborda la medición masiva del rendimiento académico de una comunidad científica tan amplia -y tan poco conocida a estos efectos- como son las áreas humanísticas y sociales, hay que asumir la presencia de errores; y más teniendo en cuenta que Google Scholar no ejerce ningún control ni normalización sobre las fuentes que procesa.

El objetivo es minimizarlos. En este sentido, debe quedar claro que la finalidad última de este producto no es reflejar exhaustiva y milimétricamente la producción académica de un investigador, sino medir con un error tolerable su rendimiento a partir de los índices h y g, a fin de conocer su fiabilidad y validez científica.

En todo caso, dada la robustez del índice $h$, poco sensible a variaciones del número de documentos y citas, estimamos que la tasa de error de los índices h y g calculados no debe ser superior el $10 \%$. Esto es, un autor con un índice $\mathrm{h}$ de 11 , tendrá un valor real que fluctuará entre 10 y 12 (de igual manera, si su índice g es de 20, su valor real deberá estar entre 18 y 22). Además, conforme el valor del índice sea más elevado (los autores más importantes), la probabilidad de error se reduce, puesto que pasar de un índice 3 a 4 es mucho más fácil o probable que pasar de un índice 13 a 14.
Tabla 1. Profesores por disciplina analizados en $H$ Index Scholar

\begin{tabular}{|l|c|c|}
\hline \multicolumn{3}{|c|}{ Ciencias sociales } \\
\hline \multicolumn{1}{|c|}{ Disciplina } & Calculados & Visualizados \\
\hline Antropología & 453 & 153 \\
\hline Ciencia política y de la administración & 442 & 142 \\
\hline Comunicación & 1.566 & 509 \\
\hline Documentación & 350 & 116 \\
\hline Economía & 8.169 & 2.742 \\
\hline Educación & 5.671 & 1.874 \\
\hline Geografía & 1.352 & 441 \\
\hline Psicología & 3.874 & 1.290 \\
\hline Sociología Total & 1.694 & 609 \\
\hline & $\mathbf{2 3 . 5 7 1}$ & $\mathbf{7 . 8 7 6}$ \\
\hline
\end{tabular}

\begin{tabular}{|l|c|c|}
\hline \multicolumn{3}{|c|}{ Ciencias jurídicas } \\
\hline \multicolumn{1}{|c|}{ Calculados } & Visualizados \\
\hline Derecho administrativo & 733 & 218 \\
\hline Derecho civil & 862 & 328 \\
\hline Derecho constitucional & 516 & 181 \\
\hline $\begin{array}{l}\text { Derecho del trabajo y seguridad } \\
\text { social }\end{array}$ & 804 & 227 \\
\hline Derecho eclesiástico del Estado & 150 & 52 \\
\hline Derecho financiero y tributario & 473 & 147 \\
\hline Derecho internacional privado & 158 & 59 \\
\hline Derecho internacional público & 334 & 109 \\
\hline Derecho mercantil & 645 & 196 \\
\hline Derecho penal & 472 & 159 \\
\hline Derecho procesal & 428 & 154 \\
\hline Derecho romano & 180 & 40 \\
\hline Filosofía del derecho & 316 & 105 \\
\hline Historia del derecho e instituciones & 220 & 79 \\
\hline Sin adscribir & 154 & 54 \\
\hline \multicolumn{1}{|c|}{ Total } & $\mathbf{6 . 4 4 5}$ & $\mathbf{2 . 1 0 8}$ \\
\hline
\end{tabular}

\begin{tabular}{|c|c|c|}
\hline \multicolumn{3}{|c|}{ Ciencias humanas } \\
\hline Disciplina & Calculados & Visualizados \\
\hline Estudios árabes, hebreos y orientales & 185 & 62 \\
\hline Filología clásica & 510 & 157 \\
\hline Filologías hispánicas & 1.678 & 572 \\
\hline Filologías modernas & 2.565 & 864 \\
\hline Filosofía & 694 & 234 \\
\hline Historia & 3.545 & 1.167 \\
\hline Lingüística general & 216 & 73 \\
\hline $\begin{array}{l}\text { Historia de la literatura y literatura } \\
\text { comparada }\end{array}$ & 131 & 44 \\
\hline Traducción e interpretación & 487 & 159 \\
\hline Total & 10.011 & 3.332 \\
\hline
\end{tabular}

\begin{tabular}{|c|c|c|}
\hline \multicolumn{3}{|c|}{ Arte } \\
\hline Área & Calculados & Visualizados \\
\hline Dibujo & 286 & 47 \\
\hline Escultura & 137 & 22 \\
\hline Música & 164 & 45 \\
\hline Pintura & 299 & 63 \\
\hline Sin adscribir & 80 & 25 \\
\hline Total & 966 & 202 \\
\hline
\end{tabular}


Por ello, y a pesar de los problemas técnicos y metodológicos que pueda presentar Google Scholar como fuente de información (errores en la identificación de documentos y citas, existencia de documentos y citas duplicados, carencia de control y normalización de sus registros), debidos al procesamiento automático que realizan sus robots, estimamos que no afectan en lo sustancial a los índices h y g ofrecidos, estando dentro de la tasa de error del $10 \%$ anteriormente señalada.

Asimismo conviene recalcar que los índices aquí mostrados no reflejan los que actualmente puedan tener los profesores, ya que las búsquedas en Google Scholar se han ejecutado en distintas fechas; en algunos casos tienen más de un año de antigüedad. Por consiguiente, deben tomarse estos datos con la cautela debida y, en ningún caso, con fines evaluativos.

\section{Notas}

1. http://hindexscholar.com

2. INE. Estadística de enseñanza universitaria. http://www.ine.es/jaxi/menu.do?type=pcaxis\&path=\%2Ft1 $3 \% 2 F p 405 \&$ file $=$ inebase $\& L=0$

3. http://www.harzing.com

4.http://roar.eprints.org/cgi/roar_search/advanced?location_ country=es

5. http://repositories.webometrics.info/en/Europe/Spain

6. http://ec3.ugr.es

7. $h t t p: / / h i n d e x s c h o l a r . c o m / c o n t a c t o$

\section{Agradecimientos}

1) Agradecemos el inestimable trabajo de recogida de datos llevado a cabo por Diego Marcos-Cartagena y Juan-Manuel Ayllón-Millán.

2) Trabajo financiado con cargo al proyecto HAR201130383-C02-02 de la Dirección General de Investigación y Gestión del Plan Nacional de I+D+I. Ministerio de Economía y Competitividad.

\section{Referencias bibliográficas}

Aguillo, Isidro F.; Ortega, José-Luis; Fernández-Pérez, Mario; Utrilla, Ana-María (2010). "Indicators for a webometric ranking of open access repositories". Scientometrics, v. 82, n. 3, pp. 477-486.

http://dx.doi.org/10.1007/s11192-010-0183-y

Archambault, Eric; Larivière, Vincent (2010). "The limits of bibliometrics for the analysis of the social sciences and humanities literature". World social science report: competing in the knowledge society. Unesco, pp. 251-254.

Archambault, Eric; Vignola-Gagné, Étienne; Côtè, Grégoire; Larivière, Vincent; Gingras, Yves (2006). "Benchmarking scientific output in the social sciences and humanities: The limits of existing databases". Scientometrics, v. 68, n. 3, pp. 329-342. http://dx.doi.org/10.1007/s11192-006-0115-z

Ardanuy, Jordi (2009). "Els estudis bibliomètrics basats en l'anàlisi de citacions en àrees d'humanitats en el període
1959-2008". BiD: textos universitaris de biblioteconomia $i$ documentació, n. 22.

http://bid.ub.edu/22/ardanuy.htm

Ardanuy, Jordi (2013). "Sixty years of citation analysis studies in the humanities (1951-2010)". Journal of the American Society for Information Science and Technology, v. 64, n. 8, pp. 1751-1755.

http://dx.doi.org/10.1002/asi.22835

Broadus, Robert N. (1971). "The literature of the social science: a survey of citation studies". International social science journal, n. 23, pp. 236-243.

Callon, Michel; Courtial, Jean-Pierre; Penan, Hervé (1995). Cienciometría. El estudio cuantitativo de la actividad científica: de la bibliometría a la vigilancia tecnológica. Gijón: Trea.

Cronin, Blaise (1984). The citation process. The role and significance of citations in scientific communication. London: Taylor Graham.

Delgado-López-Cózar, Emilio; Ruiz-Pérez, Rafael (2009). “La comunicación y edicion científica fundamentos conceptuales". En: Homenaje a Isabel de Torres Ramírez: Estudios de documentación dedicados a su memoria. Granada: Editorial Universidad de Granada, pp. 131-150.

Egghe, Leo (2006). "Theory and practice of the g-index". Scientometrics, v. 69, n. 1, pp. 131-152.

http://dx.doi.org/10.1007/s11192-006-0144-7

Giménez-Toledo, Elea; Román-Román, Adelaida (2009). “Assessment of humanities and social sciences monographs through their publishers: A review and a study towards a model of evaluation". Research evaluation, v. 18, n. 3, pp. 201-213. http://dx.doi.org/10.3152/095820209X471986

Gorraiz, Juan; Purnell, Philip J.; Glänzel, Wolfang (2013). "Opportunities for and limitations of the Book Citation Index". Journal of the American Society for Information Science and Technology, v. 64, n. 7, pp. 1388-1398.

http://dx.doi.org/10.1002/asi.22875

Hicks, Diana M. (2004). "The four literatures of social science". En: Moed, H. F.; Glänzel, W.; Schmoch, U. (Eds.). Handbook of quantitative science and technology research: The use of publication and patent statistics in studies of S\&T systems. Kluwer Academic Publishers, Netherlands, pp. 473-496.

Hicks, Diana M.; Wang, Jian (2009). “Towards a bibliometric database for the social sciences and humanities" [report]. http://works.bepress.com/diana_hicks/18

Hirsch, Jorge E. (2005). "An index to quantify an individual's scientific research output". PNAS, v. 102, n. 46, pp. 1656916572.

http://arxiv.org/abs/physics/0508025

http://dx.doi.org/10.1073/pnas.0507655102

Jacsó, Péter (2008a), "Google Scholar revisited". Online information review, v. 32, n. 1, pp. 102-114. http://dx.doi.org/10.1108/14684520810866010

Jacsó, Péter (2008b). "The pros and cons of computing the h-index using Google Scholar". Online information review, v. 32, n. 3, pp. 437-52. 
http://dx.doi.org/10.1108/14684520810889718

Jacsó, Péter (2012). “Using Google Scholar for journal impact factors and the h-index in nationwide publishing assessments in academia - siren songs and air-raid sirens". Online information review, v. 36, n. 3, pp. 462-478.

http://dx.doi.org/10.1108/14684521211241503

Kousha, Kayvan; Thelwall, Mike (2007). “Google Scholar Citations and Google Web/URL citations: a multidiscipline exploratory analysis". Journal of the American Society for Information Science and Technology, v. 58, n. 7, pp. 10551065.

http://dx.doi.org/10.1002/asi.20584

Kousha, Kayvan; Thelwall, Mike (2008). "Sources of Google Scholar citations outside the Science Citation Index: A comparison between four science disciplines". Scientometrics, v. 74, n. 2, pp. 273-294.

http://dx.doi.org/10.1007/s11192-008-0217-x

Kousha, Kayvan; Thelwall, Mike; Rezaie, Somayeh (2011). "Assessing the citation impact of books: The role of Google Books, Google Scholar, and Scopus". Journal of the American Society for Information Science and Technology, v. 62, n. 11, pp. 2147-2164.

http://dx.doi.org/10.1002/asi.21608

Leydesdorff, Loet; Felt, Ulrike (2012). "Edited volumes, monographs and book chapters in the Book Citation Index $(\mathrm{BKCl})$ and Science Citation Index ( $\mathrm{SCl}, \mathrm{SoSCl}, \mathrm{A} \& \mathrm{HCl})$ ). Journal of scientometric research, v. 1, n. 1, pp. 28-34.

Moed, Henk F. (2005). Citation analysis in research evaluation. Dordrecht: Springer.

Nederhof, Anton J. (2006). "Bibliometric monitoring of research performance in the social sciences and the humanities: a review". Scientometrics, v. 66, n. 1, pp. 81-100. http://dx.doi.org/10.1007/s11192-006-0007-2

Orduña-Malea, Enrique (2012). Propuesta de un modelo de análisis redinformétrico multinivel para el estudio sistémico de las universidades españolas. Valencia: Universidad Politécnica de Valencia [tesis doctoral].
Orduña-Malea, Enrique; Ontalba-Ruipérez, José-Antonio (2013). "Proposal for a multilevel university cybermetric analysis model". Scientometrics, v. 95, n. 3, pp. 863-884.

http://dx.doi.org/10.1007/s11192-012-0868-5

Orduña-Malea, Enrique; Serrano-Cobos, Jorge; Lloret-Romero, Nuria (2009). "Las universidades públicas españolas en Google Scholar; presencia y evolución de su publicación académica web". El profesional de la información, v. 18, n. 5, pp. 493-500.

http://eprints.rclis.org/17211

http://dx.doi.org/10.3145/epi.2009.sep.02

Osca-Lluch, Julia; Veyrat, Ana; Morales, Jesús (2013). “El consumo de información en Humanidades". Arbor, v. 189, n. 760. http://dx.doi.org/10.3989/arbor.2013.760n2012

Thelwall, Mike (2002). "Research dissemination and invocation on the web". Online information review, v. 26, n. 6, pp. 413-420.

http://dx.doi.org/10.1108/14684520210452745

Torres-Salinas, Daniel; Delgado-López-Cózar, Emilio (2013). "Cobertura de las editoriales científicas del Book Citation Index en ciencias sociales y humanidades: ¿̇la historia se repite?". Anuario ThinkEPI, v. 7, pp. 110-113.

Torres-Salinas, Daniel; Ruiz-Pérez, Rafael; Delgado-LópezCózar, Emilio (2009). "Google Scholar como herramienta para la evaluación científica". El profesional de la información, v. 18, n. 5, pp. 501-510.

http://dx.doi.org/10.3145/epi.2009.sep.03

Van Impe, Steven; Rousseau, Ronald (2006). “Web-to-print citations and the humanities". Information-Wissenschaft und Praxis, v. 57, n. 8, pp. 422-426.

White, Howard D.; Boell, Sebastian K.; Yu, Hairong; Davis, Mari; Wilson, Concepción S.; Cole, Fletcher T. H. (2009). "Libcitations: A measure for comparative assessment of book publications in the humanities and social sciences". Journal of the American Society for Information Science and Technology, v. 60, n. 6, pp. 1083-1096.

http://dx.doi.org/10.1002/asi.21045

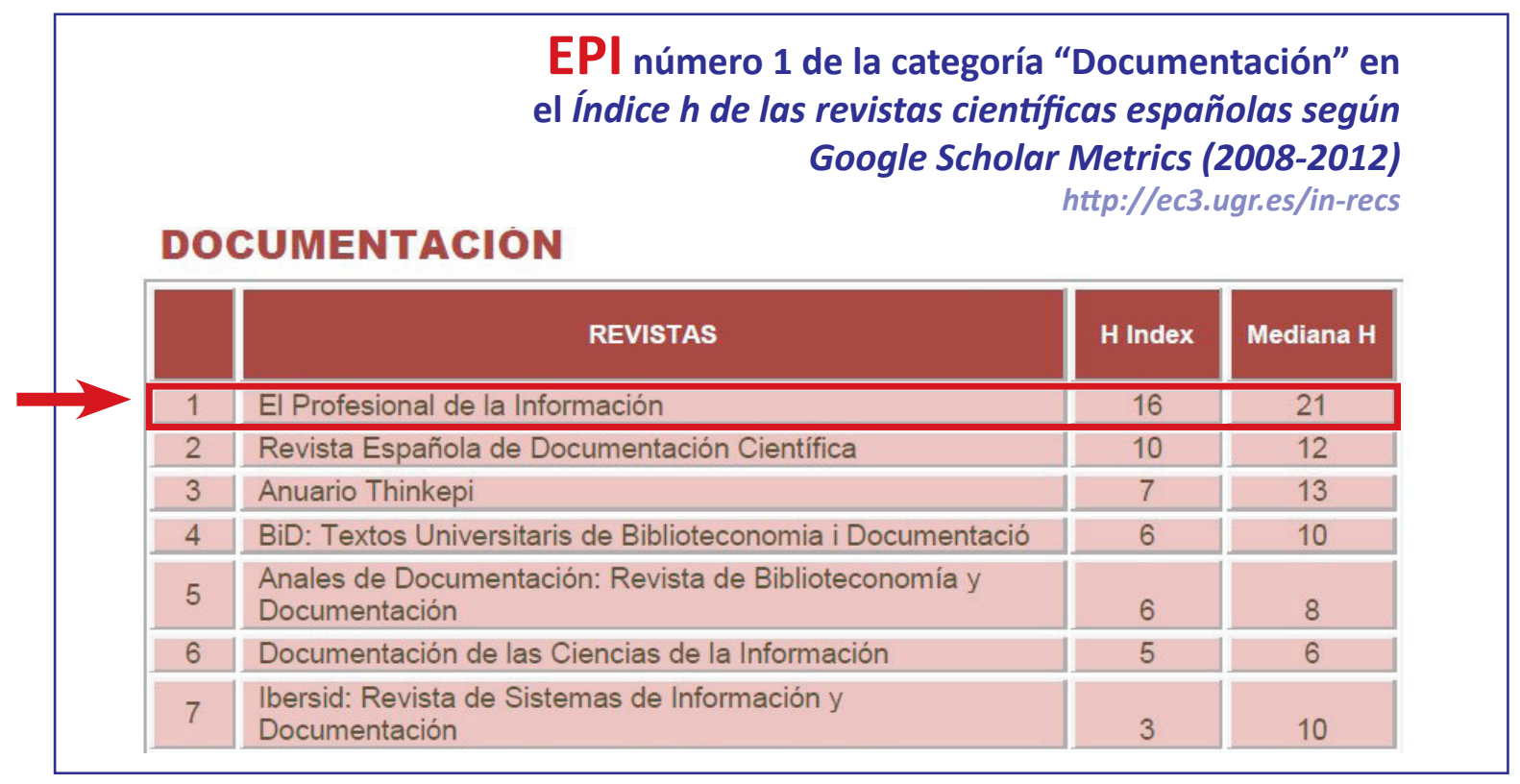

